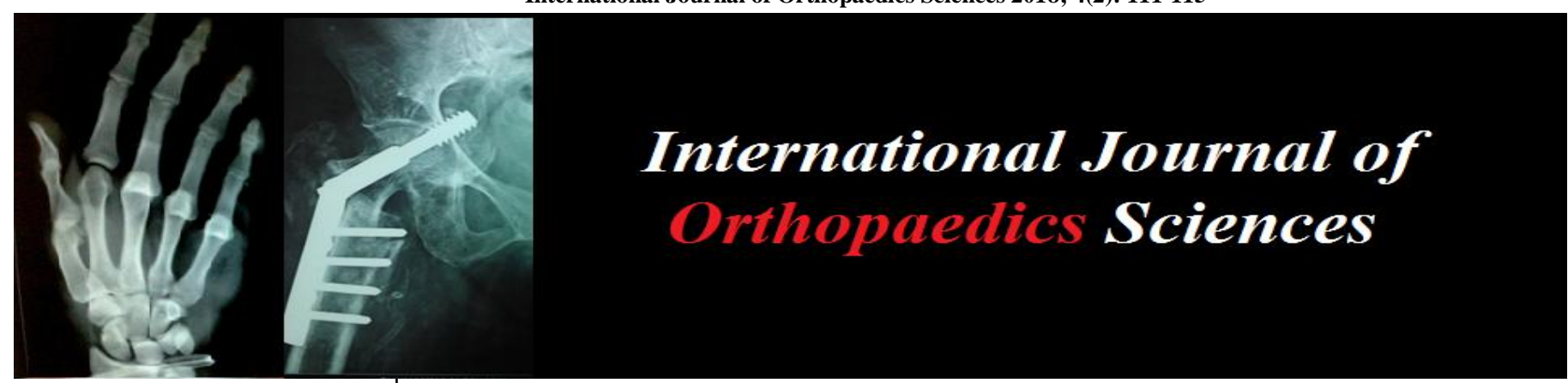

ISSN: $2395-1958$

IJOS 2018; 4(2): 111-115

(C) 2018 IJOS

www.orthopaper.com

Received: 22-02-2018

Accepted: 26-03-2018

NS Rajarajan

ESIC Mediacal College \&

PGIMSR KK Nagar, Chennai,

Tamil Nadu, India
Correspondence

NS Rajarajan

ESIC Mediacal College \&

PGIMSR KK Nagar, Chennai,

Tamil Nadu, India

\section{A comparative study of treatment of unstable intertrochanteric fractures with PFN and cemented hemiarthroplasty}

\section{NS Rajarajan}

DOI: https://doi.org/10.22271/ortho.2018.v4.i2b.19

Abstract

Aim: Aim of the study is to analyse and compare the functional outcomes and radiological results of two different surgical modalities, PFN and cemented hemiarthroplasty in the treatment of unstable intertrochanteric fractures in elderly patients.

Background: Elderly patients with intertrochanteric fractures should be mobilized early to reduce the morbidity and mortality. While cemented hemiarthroplasty allows early weight bearing, the prolonged surgical time increase the blood loss and mortality, whereas with PFN once the fracture becomes stable allows early mobilization thus overcoming the problems of recumbency. PFN efficiently preserves the femoral head and the abductor mechanism and thus has the biomechanical advantage of increased range of hip joint movements and better functional outcome.

Materials and methods: Sixty patients of age 60 years and above with comminuted intertrochanteric fractures treated by PFN or cemented hemiarthroplasty were taken up for the prospective study in our institute between january2013 and june 2015. 30 patients underwent PFN and 30 patients had hemiarthroplasty. Average period of follow up was 2 years. Post-operative complications and mortality rates were compared between the two groups. Functional results and the hip range of movements were assessed by Harris Hip Score (HHS). In the mean follow up period of 22 months radiological examination was done to assess the results \& complications.

Results: The demographic and background data of the patients in the two groups are statistically similar. At 2 years follow up PFN group patients had a mean HHS of 85.89 and HA group had 78.11. Pain, severity and range of hip movements are better in PFN group than HA group. Radiologicaly 4 patients in HA group had stem loosening and 2 patients had screw cut out in the PFN group with subsequent pain in the final follow up.

Conclusion: PFN is a better option for the treatment of elderly intertrochanteric fractures as the operative time is short, easier surgical procedure \& has less mortality. PFN also gives a better functional outcome in the late follow up than hemiarthoplasty.

Keywords: Internal fixation, prosthesis, walking ability, elderly patients, trochanteric fractures

\section{Introduction}

Intertrochanteric fractures disable further the already incapacitated elderly patients. Intertrochanteric fractures increases the morbitity and mortality chances in elderly patients because of lack of mobility ${ }^{[1,2]}$. Intertrochanteric fractures has become a very common problem nowadays in elderly patients because of increased life expectancy and osteoporosis ${ }^{[3,4]}$. Aim of any treatment should be to mobilize these patients early. To achieve this anatomical reduction of comminuted fracture and stable fixation by PFN will restore the neck shaft angle and preserve the natural hip joint. Because of this advantage patients have less pain and could walk better postoperatively and can be rehabilitated better and early. Because of some demeritis of PFN, hemiarthroplasty has become an effective alternative method of treatment of comminuted intertrochanteric fractures which allows early weight bearing and rehabilitation and addresses the demeritis of PFN. But HA has its own disadvantage.

Internal fixation of stable fractures is easy to perform and gives good results. Anatomical reduction of unstable fractures (Evans type 3 or 4 ) and its maintenance by internal fixation is difficult due to comminuted fracture geometry and poor bone quality ${ }^{[4,5]}$. 
Fracture collapse, screw pull out, screw cut out. Etc are common in internal fixation ${ }^{[6,7]}$

To overcome this complication proximal femoral nail antirotation (PFNA) intra medullary fixation implant with additional antirotation stability was introduced by AO/ASIF [8]. For unstable intertrochanteric fractures PFN is biomechanically superior as it shares the load and control excessive shear force ${ }^{[9-12]}$.

On the basis of this we made a prospective study of the functional outcomes of the patients with unstable intertrochanteric fractures subjecting them to any one of the two surgical methods and for analysing the efficacy and superiority between these two techniques in terms of clinical and radiological results.

\section{Materials and Methods}

Sixty patients of age between 60 to 85 years with unstable intertrochanteric fractures (Evans type 3 or 4) who had underwent PFN or hemiarthroplasty surgeries between January 2013 and June 2015 were taken up for this prospective study.

Patients with underlying comorbidities and were independently walking before the fracture were included in the study. Patients above 85 years of age, with stable intertrochanteric fractures and patients who were less active before the fracture were excluded.

Informed written consent was taken from the patients who met the inclusion criteria. These patients were assigned either to the PFN group or to the hemiarthroplasty group randomly. In first group of 30 patients, 12 were males and 18 females and all of them underwent proximal femoral nailing (PFN). In the other group of 30 patients 13 were males and 17 females and all of them underwent hemiarthroplasty (HA). The choice of the surgical method either PFN or HA, for a given Intertrochanteric fracture were consecutively and alternatively allotted in the randomization process. Proximal femoral nailing and cemented hemiarthroplasty are the routine surgical procedures in our institute for intertrochanteric fractures and all operated cases were followed up for a period of 2 years.

Demographic and preoperative data including age, gender and fracture type according to Evans classification were recorded before the procedure along with the postoperative data including duration of hospital stay, blood loss, time of bed side mobilization, time taken for full weight bearing. Postoperative complications and mortality were recorded in the follow up. Patients were taken up for surgery 48 to 72 hours after the fracture, after obtaining anaesthetic fitness.

In the proximal femoral nailing procedure the comminuted fracture fragments were reduced by closed methods or if required by open methods to obtain a satisfactory reduction, so as to restore a normal neck shaft angle. In adequate reduction valgus angulation was accepted. Standard $240 \mathrm{~mm}$ nail was used. Proximally $8 \mathrm{~mm}$ and $6 \mathrm{~mm}$ PFN screws were used and distally $4.5 \mathrm{~mm}$ cortical screws were used for locking. (Fig $1 \& 2$ ).

In hemiarthroplasty technique great care was taken to retain the comminuted fracture fragments as much as possible, mainly the greater trochanter and the calcar, so that the abductor mechanism is restored and the prosthesis sinking can be prevented by the metaphyseal stability. Modular bipolar prosthesis was implanted with bone cement.

Patients with hemiarthroplasty prosthesis were allowed full weight bearing on the 2 nd post-operative day. Patients with PFN were rehabilitated non- weight bearing from 3rd post- operative day. Full weight bearing without support was allowed after the 7th week in PFN group and after 6th week in hemiarthroplasty group.

Clinical and radiological evaluations of the patients were done in the follow up period of 3 months, 6 months \& then yearly up to 2 years. Clinical and functional assessment was done using Harris Hip Score (HHS). In radiological assessment of PFN group any screw cut or pull out was noted. In HA group, stem subsidence more than $3 \mathrm{~mm}$ or a persistent radiolucent line of $2 \mathrm{~mm}$ or more at bone cement interface was considered as stem loosening.

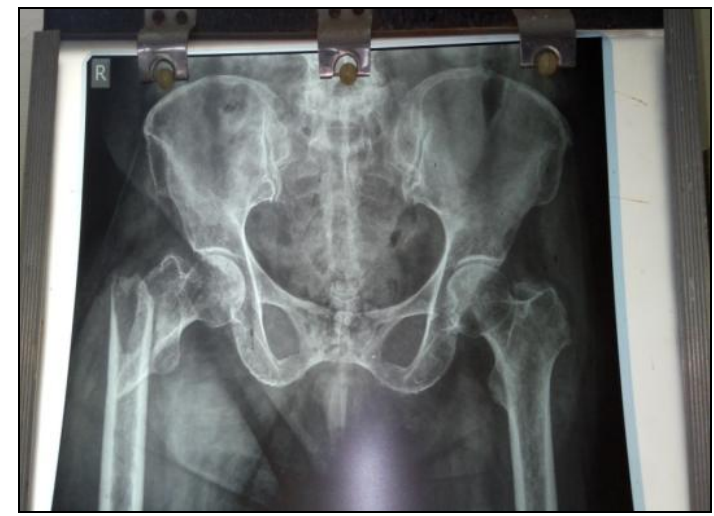

Fig 1: Intertrochanter Fracture-Pre Op

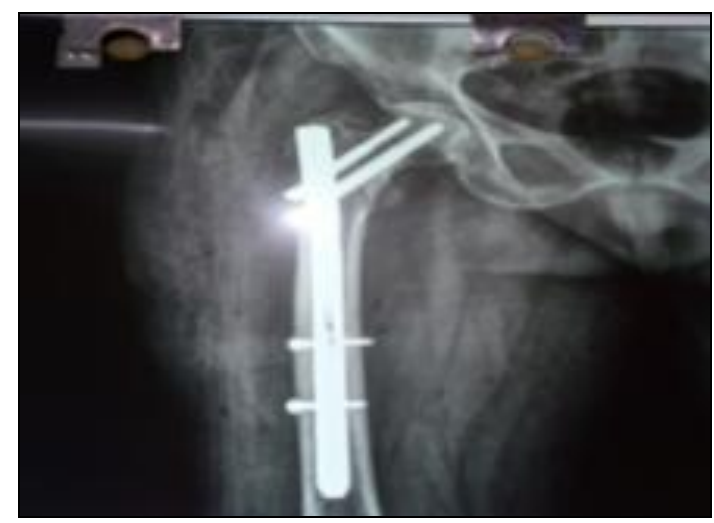

Fig 2: Intertrochanteric fracture post $\mathrm{Op}$

\section{Statistical Analysis}

The Harris Hip scores were assessed for all the patients at 3 months, 6 months, 1 year and 2 years following the surgeries. These scoresw ere compared using the repeated measures ANOVA test with the two surgery groups entered as independent variable. Further, the Bed side mobilization (BSM) and the Independent walking (IW) were also compared between the two surgery groups using independent sample t test. Statistical significance value were set at $p<0.05$.

\section{Results}

Sixty elderly patients with unstable intertrochanteric fractures have been included in our study. In PFN group of 30 patients 12 were male and 18 were female. Average age was 74.1 (range 63-85 years). 10 patients had Evans type 3 fractures and 20 patients had Evans type 4. In HA group of 30 patients 13 were males and 17 were females. Average age was 72 (range 60-83 years). 13 patients had Evans type 3 fractures and 17 patients had Evans type 4 fractures. In HA group 3 patients and in PFN group 1 patient expired after 1 year. One patient in each group has not turned up for follow up after 18 months. Hence the functional assessment at the final follow up of 2 years was derived from 54 patients. The mean follow 
up was in PFN group 22.5 (range 12 to 24 months) and in HA group 21.66 (range 12 to 24 months).

HA group patients had higher HHS than the PFN group patients in the initial follow up of 3rd month 70vs64.89 and 6th month 72.38vs67.32 with statistically significant difference $(p<0.0001)$. However PFN group patients had better HHS than the HA group patients in the later follow up of $1 \mathrm{st}$ year $79.78 \mathrm{vs} 76.69$ and 2 nd year $85.89 \mathrm{vs} 78.11$ significant difference were observed $(p<0.0001)$ statistically. PFN Group had poor HHS in the early follow up and good HHS in the 2nd year follow up. HA group had fair HHS in the latter follow up period. HHS is depicted in Table -1 and flow chart 1 .

HA group patients were allowed full weight bearing on 2nd post-operative day. PFN group patients were mobilized bed side non-weight bearing at mean 4.1 days (range 3-6 days) $(p<0.0001)$. Independent walking without support and return of patients to routine daily activities was earlier in HA group mean 6.6 weeks (range 6-8 weeks) than in the PFN group mean 7.6 weeks (range 6-10 weeks) $(p<0.0001)$. Limb length discrepancy was significant in HA group with 4 patients had limb lengthening $>2 \mathrm{~cm}$ and 2 patients had shortening $>2 \mathrm{~cm}$. In PFN group one patient had $>2 \mathrm{~cm}$ shortening because of varus collapse. Clinical studies results are represented in Table -2/flow chart-2.

Two patients in HA group developed deep infection for which implant removal and excision arthroplasty was done as they were not fit for revision surgery. These two patients died after one year. Screw cut out was observed in 2 patients in PFN group. One patient had revision THR but he expired in the post-operative period. The other patient had not turned up for follow up. Stem loosening and implant subsidence occurred in 4 patients in HA group (Fig: 3). Three of these patients have become less active and had decreased functional out come in the follow up. Revision surgery was deferred in these patients due to associated co morbidities. The other patient has not turned up for follow up. In one patient while broaching the femur got fractured and was fixed by circlage wiring (Fig: 4). Complications are depicted in Table -3 .

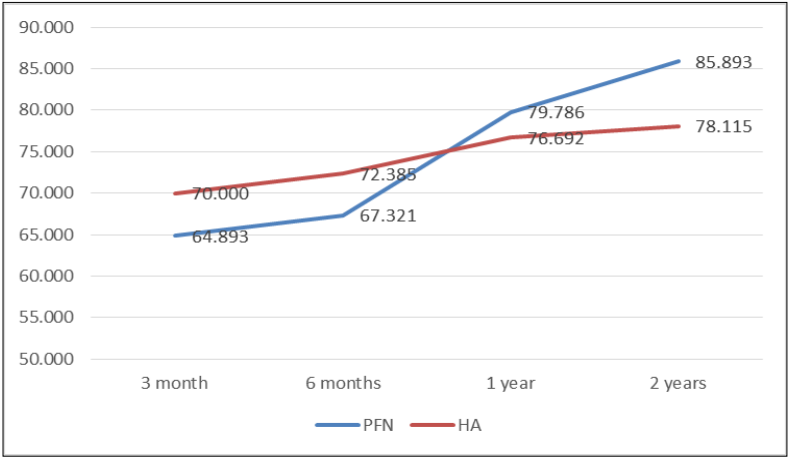

Chart 1: Haris Hip Scores at post surgical follow - up
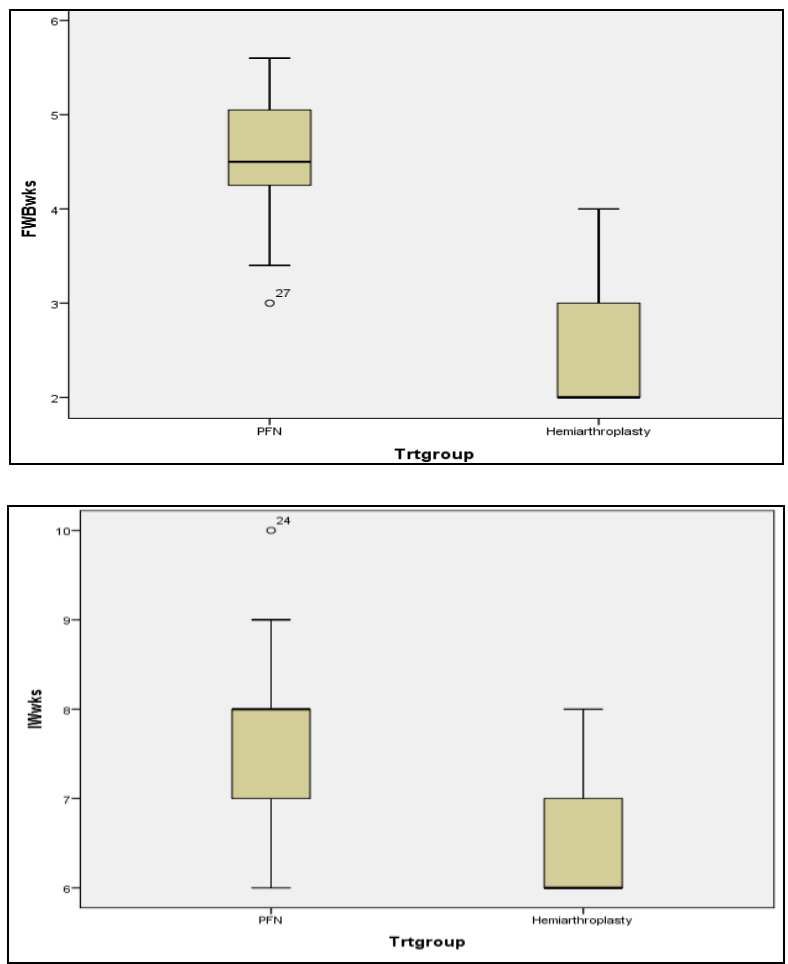

Chart 2

Table 1: The Mean Haris Hip Scores at the various time periods following the surgery

\begin{tabular}{|c|c|c|c|c|c|}
\hline \multirow{2}{*}{ Treatment } & \multirow{2}{*}{ Time of HHS measurement } & \multirow{2}{*}{ Mean } & \multirow{2}{*}{ Std. Error } & \multicolumn{2}{|c|}{ 95\% Confidence Interval } \\
\cline { 2 - 6 } & & & \multicolumn{2}{|c|}{ Lower Bound } & Upper Bound \\
\hline \multirow{3}{*}{ PFN } & 3 months & 64.893 & 1.229 & 62.427 & 67.359 \\
\cline { 2 - 6 } & 6 months & 67.321 & 1.171 & 64.972 & 69.671 \\
\cline { 2 - 6 } & 1 year & 89.786 & 1.163 & 77.453 & 82.119 \\
\hline \multirow{3}{*}{ HA } & 2 years & 70.000 & 1.275 & 67.441 & 72.559 \\
\cline { 2 - 6 } & 3 months & 72.385 & 1.215 & 69.946 & 74.823 \\
\cline { 2 - 6 } & 6 months & 76.692 & 1.207 & 74.271 & 79.113 \\
\cline { 2 - 6 } & 1 year & 78.115 & 1.166 & 75.776 & 80.455 \\
\hline
\end{tabular}

Table 2: Difference between two groups with respect to Bed side mobilization and Independent Walking

\begin{tabular}{|c|c|c|c|}
\hline Treatment Group & Mean Days for Bed side mobilization & Mean weeks for Independent walking & p value \\
\hline PFN & 4.55 & 7.68 & $<0.001$ \\
\hline HA & 2.31 & 6.54 & $<0.001$ \\
\hline
\end{tabular}

Table3: Complications related to surgery

\begin{tabular}{|c|c|c|c|}
\hline & PFN & HA & P-Value \\
\hline Infection & & & \\
\hline Superficial & $2(6.6 \%)$ & $3(10 \%)$ & $P<0.001$ \\
\hline Deep & - & $2(6.6 \%)$ & $P<0.001$ \\
\hline Pulmonary & $2(6.6 \%)$ & $1(3.3 \%)$ & $P<0.001$ \\
\hline Pressure sores & $1(3.3 \%)$ & $3(10 \%)$ & $P<0.001$ \\
\hline Implant failure & $2(6.6 \%)$ & $4(13.3 \%)$ & $P<0.001$ \\
\hline
\end{tabular}




\begin{tabular}{|c|c|c|c|}
\hline Revision surgery & $1(3.3 \%)$ & $2(6.6 \%)$ & $P<0.001$ \\
\hline Mortality rate (2yrs) & $1(3.3 \%)$ & $3(10 \%)$ & $P<0.001$ \\
\hline
\end{tabular}

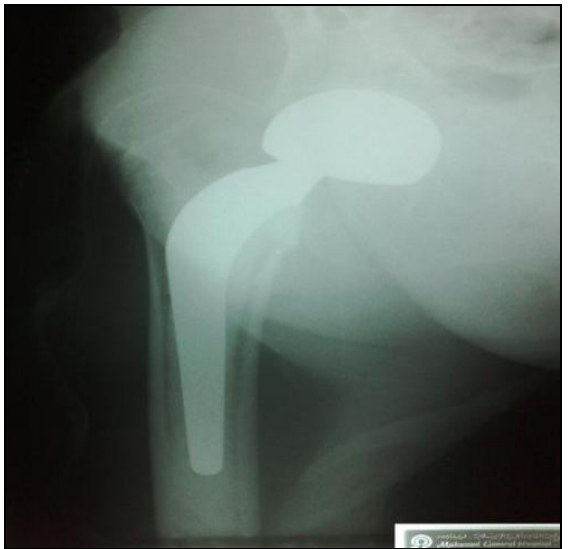

Fig 3: Stem Loosening

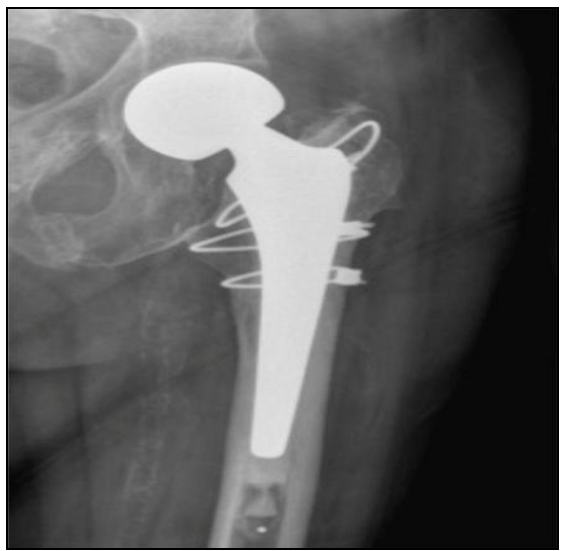

Fig 4: per op femoral stem fracture

\section{Discussion}

Aim of the treatment of the elderly patients with intertrochanteric fractures is to mobilize the patients early so as to reduce the morbidity and mortality. Post operatively patients should not have any gross disability so that they can have the same functional level as before the fracture. Long term medical complications can be prevented if the patient is rehabilitated satisfactorily ${ }^{[13-15]}$.

For elderly patients with comorbid factors the preferred surgical technique should be minimally invasive with less blood loss. The chosen implant should allow early full weight bearing and early rehabilitation there by the problems of prolonged recumbence can be avoided ${ }^{[11]}$.

Sinno $\mathrm{K}$ et al. and Kayali $\mathrm{C}$ et al. have reported that early weight bearing following hemiarthroplasty significantly reduces the post-operative complications [16]. In internal fixation method weight bearing should be delayed till the $\mathrm{x}$ ray shows callus formation at the fracture site. However if the medial calcar was reduced adequately to make good opposition and internal fixation was stable, patients may be mobilized non-weight bearing with walker early thereby prolonged recumbence complications can be avoided. Patients hip joints are preserved in PFN technique thus prosthesis related complications are avoided. Friction of the metal head on already degenerated acetabular surface increases the hip joint pain which was experienced in few of the HA group patients in our series.

In our series patients in PFN group bed side mobilization without weight bearing with walker support was started at mean 4.1 days (range 3-6 days). These patients were able to walk full weight bearing without support at mean 8.6 weeks (range 6-10 weeks). The time taken for the patients in PFN group for return to daily routine activities were almost similar to that in the HA group. Anatomical reduction and stable fixation by PFN attributes for the early mobilization of these patients. Korkmaz et al. stated that in intertrochanteric fractures PFN fixation is a reliable method with good union rates and low complication rates ${ }^{[17]}$. In our series two patients in PFN group had screw cut out. In both these cases reduction was not stable. Hemiarthroplasty has the advantage of early mobilization but the subsequent follow up shows decreased functional out comes, more complications and more mortality rates than the PFN group. In our study 3 patients in HA group expired during the follow up. The bone cementation increase the intramedullary pressure which may lead to fat embolization. This effect on mortality has become a major concern ${ }^{[18-22]}$. Christie et al. reported that cementation leads to significant fat embolisim cascades than the uncemented hemiarthroplasty ${ }^{[19]}$. Donaldson et al. reported that in high risk patients uncemented hemiarthroplasty decreases the rate of morbidity and mortality ${ }^{[20]}$. A review article on 7774 hip fracture patients documented that high mortality rate was found in cemented hemiarthroplasty ${ }^{[21]}$. Hossain et al. reported in elderly patients eight case of perioperative death following cemented hemiarthroplasty ${ }^{[22]}$.

In the hemiarthroplasty technique the prosthesis metaphyseal fit should be obtained by reconstructing the comminuted calcar and the greater trochanter or by the bone cement mantle if the distorted comminuted fragments cannot be restored. Kim y et al. reported that comminuted posteromedial fragments should be stably fixed to avoid stem subsidence ${ }^{[23]}$. This complex surgical technique prolongs the surgery time and increases the blood loss and infection rate.

Parker et al. reported that primary prosthetic replacement may be an ideal method in extremely comminuted osteoporotic intertrochanteric fractures with pre-existing co morbid complications and degenerative arthritis ${ }^{[24]}$.

PFN is a closed technique, with percutaneous insertion of the nail thus lesser operative time and less blood loss which is ideal for already debilitated elderly patients. It stabilizes the fractures with subtrochanteric extension and reverse oblique fractures.

In our study 2 patients in HA group developed deep infection, while none had deep infection in the PFN group. HA group patients had better HHS than those in the PFN group in the early follow up period. However later at two years follow up the mean HHS in PFN group was 80.16 and in the HA group was 67.5. Shen $\mathrm{j}$ et al. have reported that stable reduction and rigid internal fixation of comminuted osteoporotic intertrochanteric fractures gives higher HHS, lesser postoperative pain and better walking ability than those treated with hemiarthroplasty ${ }^{[25]}$.

Hence elderly patients with multiple medical complications, who are physiologically weak and who could not with stand the early post-operative rehabilitation programme cemented hemiarthroplasty may be opted as the primary treatment of comminuted intertrochanteric fractures as it allows earlier and easier mobilization. However stable reduction and rigid internal fixation of osteoporotic comminuted intertrochanteric fractures by proximal femoral nail also allows early ambulation. In internal fixation methods, restoration of anatomical alignment of fracture fragments, maintenance of 
neck shaft angle and preservation of the femoral head allows better range of hip movements, better walking ability and better functional out comes in the latter follow up once the fracture is united. In addition these patients can sit cross legged and can squat which is difficult after hemiarthroplasty.

\section{Conclusion}

Anatomical reduction and stable fixation of unstable intertrochanteric fractures with PFN gives good functional outcomes and better ROM than hemiarthroplasty. Though hemiarthroplasty allows early weight bearing and by passes the fracture uniting time the more complex surgical technique results in increased morbidity and mortality, subsequent follow up shows stem loosening and decreased functional results.

\section{References}

1. Bartucci EJ, Gonzale MH, Cooperman DR. The effect of methl methacrylate as adjunctive on fixation and function failures in patients with intertrochanteric fractures. J Bone Joint Surg Am. 1985; 67:1094-1107.

2. Koval KJ, Zuckerman JD. Hip fractures re an increasingly important public health problem. Clin Orthop Relat Res. 1998; 348:2.

3. Koval KJ, Chen AL, Aharanoff GB, Egol KA, Zuckerman JD. Clinical pathway for hip fractures in the elderly: the Hospital for Joint Diseases experience. Clin Orthop Relat Res. 2004; 425:72-81.

4. Evans EM. The treatment of trochanteric fractures of the femur. J Bone Joint Surg Am. 1949; 31:190-203.

5. Marsh JL, Slongo TF, Agel J, Broderick JS, Creevey W, De Coster TA et al. Fracture and dislocation compendium: Orthopaedic Trauma Association classification, database and outcome committee. J Orthop Trauma, 2007, 1S1-133.

6. Bannister GC, Gibson AG, Ackroyd CE, Newman JH. The fixation and prognosis of trochanteric fractures: A randomized prospective controlled trail. Clin Orthop Relat Res. 1990; 254:242-246.

7. Moller BN, Lunct U, Grymer F, Bartholdy NJ. Trochanteric hip fractures instability following internal fixation: Radiographic comparison of the Richards sliding screw-plate and the Mcl Laughlin nail-plate. Acta Orthop Scand. 1984; 55:517-520.

8. Pu JS, Liu L, Wang GL, Fang Y, Yang TF. Results of the proximal femoral nail anti-rotation (PFNA) in elderly Chinese patients. Int Orthop. 2009; 33:1441-1444.

9. Mereddy P, Kamath S, Ramakrishnan M, Malik H, Donnachie N. The AO/ASIF proximal femoral nail antirotation (PFNA): a new design for the treatment of unstable proximal femoral fractures. Injury. 2009; 40:428-432.

10. Jones HW, Johnston P, Parker M. Are short femoral nails superior to the sliding hip screw? A meta-analysis of 24 studies involving 3,279 fractures. Int Orthop. 2006; 30:69-78.

11. Takigami I, Matsumoto K, Ohara A. Treatment of trochanteric fractures with the PFNA (proximal femoral nail antirotation) nail system: report of early results. Bull NYU Hosp Jt Dis. 2008; 66:276-279.

12. Zou J, Xu Y, Yang H. A comparison of proximal femoral nail antirotation and dynamic hip screw devices in trochanteric fractures. J Int Med Res. 2009; 37:10571064.

13. Geiger F, Zimmermann-Stenzel M, Heisel C, Lehner B,
Daecke W. Trochanteric fractures in the elderly: the influence of primary hip arthroplasty on 1-year mortality. Arch Orthop Trauma Surg. 2007; 127:959-966.

14. Kesmezacar H, Ogut T, Bilgili MG, Gokay S, Tenekecioglu Y. Treatment of intertrochanteric femur fractures in elderly patients: internal fixation or hemiarthroplasty. Acta Orthop Traumatol Turc. 2005; 39:287-294.

15. Tang P, Hu F, Shen J, Zhang L, Zhang L. Proximal femoral nail antirotation versus hemiarthroplasty: a study for the treatment of intertrochanteric fractures. Injury. 2012; 43:876-881.

16. Sinno K, Sakr M, Girard J, Khatib H. The effectiveness of primary bipolar arthroplasty in treatment of unstable intertrochanteric fractures in elderly patients. North American Journal of medical sciences. 2010; $1: 2(12): 561$.

17. Korkmaz MF, Erdem MN, Disli Z. Outcomes of trochanteric femoral fractures treated with proximal femoral nail: an analysis of 100 consecutive cases. Clin Interv Aging. 2014; 9:569-574.

18. Elmaraghy AW, Humeniuk B, Anderson GI, Schemitsch EH, Richards RR. The role of methyl methacrylate monomer in the formation and haemodynamic outcome of pulmonary fat emboli. J Bone Joint Surg Br. 1998; 80:156-61.

19. Christie J, Burnett R, Potts HR, Pell AC. Echocardiography of transatrial embolism during cemented and uncemented hemiarthroplasty of the hip. J Bone Joint Surg Br. 1994; 76:409-12.

20. Donaldson AJ, Thomson HE, Harper NJ, Kenny NW. Bone cement implantation syndrome. Br J Anaesth. 2009; 102:12-22.

21. Parvizi J, Ereth MH, Lewallen DG. Thirty-day mortality following hip arthroplasty for acute fracture. J Bone Joint Surg Am. 2004; 86-A:1983-8.

22. Hossain M, Andrew JG. Is there a difference in perioperative mortality between cemented and uncemented implants in hip fracture surgery, injury. 2012; 43:2161-4.

23. Kim Y, Moon JK, Hwang KT, Choi IY, Kim YH. Cementless bipolar hemiarthroplasty for unstable intertrochanteric fractures in octogenarians. Acta Orthop Traumatol Turc. 2014; 48:424-430.

24. Parker MJ, Handoll HH. Replacement arthroplasty versus internal fixation for extracapsular hip fractures. Cochrane Database Syst Rev, 2000, CD000086.

25. Shen J, Wang DL, Chen GX, Yang HL, Li L, Wei MX et al. Bipolar hemiarthroplasty compared with internal fixation for unstable intertrochanteric fractures in elderly patients. J Orthop Sci. 2012; 17:722-729. 